\title{
Dimerization specificity of the leucine zipper-containing bZIP motif on DNA binding: prediction and rational design
}

\author{
Charles R. Vinson, ${ }^{1,2}$ Tsonwin Hai, ${ }^{3}$ and Susan M. Boyd ${ }^{1}$ \\ ${ }^{1}$ Laboratory of Biochemistry, National Cancer Institute, National Institutes of Health, Bethesda, Maryland 20892 USA; \\ ${ }^{3}$ Department of Medical Biochemistry and Ohio State Biotechnology Center, Ohio State University, \\ Columbus, Ohio 43210 USA
}

\begin{abstract}
We propose an interhelical salt bridge rule to explain the dimerization specificity between the two amphipathic $\alpha$-helices in the leucine zipper structure. Using the bZIP class of DNA-binding proteins as a model system, we predicted and designed novel dimerization partners. We predicted that ATF4, a member of the ATF/CREB family of transcription factors, would preferentially form heterodimers with IGEBP1, a member of the C/EBP family. We also predicted that neither ATF4 nor IGEBP1 would form heterodimers with DBP, a member of the C/EBP superfamily. These predictions were verified using a gel mobility-shift assay. To further test the value of this interhelical salt bridge rule, we modified the bZIP protein C/EBP attempting to design molecules that would form preferentially heterodimers with C/EBP or molecules that would not interact with C/EBP. These designed molecules behaved as predicted. Therefore, we conclude that this interhelical salt bridge rule is useful in understanding the dimerization specificity of bZIP proteins. In addition, we suggest that this rule could be used to design novel "dominant-negative" molecules to specifically inhibit the function of target leucine zipper proteins in vivo.
\end{abstract}

[Key Words: bZIP; C/EBP family; ATF/CREB family; DNA-binding proteins; dimerization specificity]

Received February 9, 1993; revised version accepted April 9, 1993.

In the last several years, there has been an explosion in the identification of sequence-specific DNA-binding proteins, many of which must dimerize for productive DNA binding. One such example is the basic leucine zipper (bZIP) class of DNA-binding proteins (Vinson et al. 1989). The dimeric bZIP proteins are characterized by a bipartite $\alpha$-helical structure: The amino-terminal half of the motif interacts with sequence-specific DNA /Agre et al. 1989; Talanian et al. 1990), whereas the carboxy-terminal half of the motif dimerizes (Gentz et al. 1989; Kouzarides and Ziff 1989b; Landschultz et al. 1989; Turner and Tjian 1989), to form a leucine zipper or coiled-coil structure (Landschultz et al. 1988; O'Shea et al. 1989a, 1991; Saudek et al. 1991). The leucine zipper motif has been shown to be a subset of the more general coiled-coil structure (O'Shea et al. 1991). The overall structure of this dimer when bound to DNA has been modeled as a Y-shaped complex (O'Neil et al. 1990; Vinson et al. 1989), an impression reinforced by the recent cocrystal of a bZIP protein bound to DNA (Ellenberger et al. 1992). The top of the Y represents two $\alpha$-helical amino-terminal basic regions that bind to DNA. The bottom, or stem, of the $\mathrm{Y}$ represents the leucine zipper

${ }^{2}$ Corresponding author. domain that brings the two DNA-binding $\alpha$-helices together.

As originally proposed by Landschultz et al. (1988), bZIP proteins not only homodimerize but also heterodimerize by the leucine zipper structure (Hai et al. 1989; Ivashkiv et al. 1990; Roman et al. 1990; Cao et al. 1991; Hai and Curran 1991; Williams et al. 1991; Schindler et al. 1992). However, only specific bZIP protein pairs can heterodimerize. Experiments using chimeric proteins and peptides indicate that the dimerization region itself contains all of the structural information needed to regulate dimerization specificity (Agre et al. 1989; Kouzarides and Ziff $1989 \mathrm{~b}$; O'Shea et al. 1989b). The structural rules that govern the specificity of two amphipathic helices forming a leucine zipper dimer are the subject of this report.

To generate a repeating helical dimerization interface in a coiled-coil, an $\alpha$-helix overtwists slightly, going from 3.6 to 3.5 amino acids per turn. This allows the structure to repeat itself after two helical turns or 7 amino acids (a heptad repeat). Figure 1 shows schematic side and end views of the coiled-coil structure /Cohen and Parry 1990) using the standard nomenclature for the 7 unique amino acid positions $(\boldsymbol{a}, \boldsymbol{b}, \boldsymbol{c}, \boldsymbol{d}, \boldsymbol{e}, \boldsymbol{f}, \boldsymbol{g})$ (Hodges et al. 1972). The $\boldsymbol{a}$ and $\boldsymbol{d}$ residues pack in a regular "knobs and holes" pattern along the dimerization interface to 

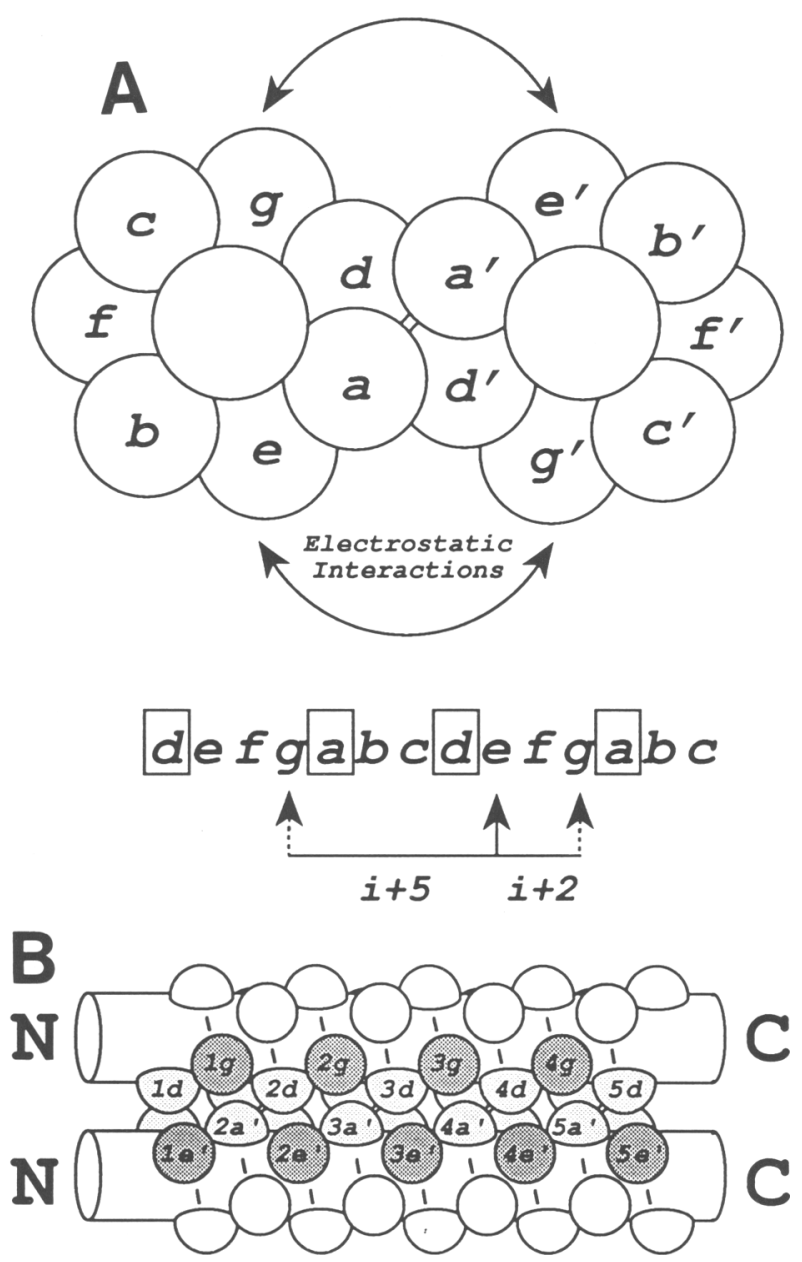

Figure 1. End and side views of a coiled-coil structure. $(A)$ The letters inside the circles represent standard nomenclature for the 7 amino acids found in unique positions in a coiled-coil. The view is from the amino terminus looking into the paper. Amino acids at the $\boldsymbol{a}$ and $\boldsymbol{d}$ positions create a hydrophobic core between the interacting helices. The electrostatic interactions hypothesized to occur between amino acids at the $\boldsymbol{e}$ and $\mathbf{g}$ positions are noted. The bottom of the end view shows the two different types of interhelical salt bridges predicted from model building. $i+5$ refers to the interaction between the amino acid at the $g$ position of one helix and the amino acid at the $\boldsymbol{e}^{\prime}$ position 5 amino acids carboxy-terminal on the opposite helix. $i+2$ refers to the interaction between the amino acid at the $e$ position from one helix and the amino acid at the $\boldsymbol{g}^{\prime}$ position 2 amino acids carboxy-terminal on the opposite helix. The $i+5$ and $i+2$ nomenclature follows the convention used to describe intrahelical salt bridges (Marqusee and Baldwin 1987). (B) This view (Hu et al. 1990) shows all of the amino acids that are depicted in Fig. 2, with the numbering relative to the first leucine of the dimerization region. The proposed $i+5$ interaction between the $g$ and the following $\boldsymbol{e}^{\prime}$, as well as the i +2 interaction between the $\boldsymbol{e}^{\prime}$ and the following $g$ position, can be visualized. The supercoiling of the two helices around each other is not depicted. With supercoiling, the $i+5$ interaction is closer than the $i+2$ interaction.

create the hydrophobic core that is critical for dimerization. The $\boldsymbol{e}$ and $\boldsymbol{g}$ positions that flank the dimerization interface contain a large number of charged amino acids and are thought to interact electrostatically to form salt bridges between helices (Cohen and Parry 1990). This notion is supported by the mutagenesis studies of the GCN4 coiled-coil region by Kim and colleagues $\left(\mathrm{O}^{\prime}\right.$ Shea et al. 1992). They demonstrated that when all of the $\boldsymbol{e}$ and $g$ positions of GCN4 are changed to the corresponding amino acids from Fos and Jun, the chimeric peptides heterodimerize just as Fos and Jun do.

To investigate how $\boldsymbol{e}$ and $\boldsymbol{g}$ residues determine leucine zipper dimer specificity, we resorted to model building. Figure 1 shows the two possible types of interhelical salt bridges suggested by model building. One interaction is between the $\boldsymbol{g}$ position of one helix and the following $\boldsymbol{e}$ position on the opposite helix, 5 amino acids carboxyterminal (designated $\boldsymbol{e}^{\prime}$ ). The second possible interaction is between the $\boldsymbol{e}$ position and the following $\boldsymbol{g}$ position on the opposite helix, 2 amino acids carboxy-terminal (designated $g^{\prime}$ ). Following the convention used to identify intrahelical salt bridges (Marqusee and Baldwin 1987), we refer to these different structural configurations of interhelical salt bridges as $i+5$ and $i+2$ salt bridges, respectively.

The $i+5$ salt bridge configuration was observed in the dimerization of one leucine zipper protein, GCN4. The $\mathrm{X}$-ray structure of the GCN4 leucine zipper (O'Shea et al. 1991) and GCN4 complexed to DNA (Ellenberger et al. 1992) shows three interhelical i 5 salt bridges. A fourth expected interaction was not observed; instead, the charged side chain of the fourth $i+5$ pair was involved in crystal-packing interactions. In a nuclear magnetic resonance structure of the GCN4 dimerization region (Saudek et al. 1991), it was not possible to determine the positions of the $\boldsymbol{e}$ and $\boldsymbol{g}$ side chains, suggesting that these side chains are locally unstructured in solution. The $i+5$ salt bridge has been suggested to be important for the dimerization of another coiled-coil protein, tropomyosin. McLachlan and Stewart (1975) noticed a large number of complementary charges in the $i+5$ position when they examined the tropomyosin sequence. They suggested that these interhelical electrostatic interactions are important for the unstaggered dimerization of tropomyosin.

We attempted to predict novel dimerization partners among the known mammalian bZIP proteins to test whether the interhelical $i+5$ electrostatic interactions are important for specifying bZIP dimerization. Our predictions were tested and verified. Encouraged by our success, we modified the bZIP protein, CCAAT/enhancingbinding protein (C/EBP), to create a molecule that would preferentially heterodimerize with C/EBP and a molecule that would not heterodimerize with C/EBP. The designed molecules behaved as predicted. These results suggest that the $i+5$ salt bridge rule can be used as a general structural rule to predict and design novel dimerization partners among bZIP proteins.

\section{Results}

Potential salt bridges in bZIP dimerization

Sequences for $>20$ members of the bZIP class of tran- 
scription factors from mammalian systems have been published, and the number is continually growing. To date, very little is known about many of these molecules except that they bind DNA with some sequence specificity. Figure 2 shows the protein sequence of the dimerization region of 20 mammalian bZIP proteins grouped into three general families depending on the limited knowledge of the DNA sequences that they bind: C/EBP, Fos/Jun, and activating transcription factor (ATF) [or cAMP response element binding (CREB)] families. We have examined these sequences to determine whether we can rationalize the known dimerization properties of these molecules by considering electrostatic interactions. In the sequences presented in Figure 2 , more than two-thirds of the $\boldsymbol{g}$ and $\boldsymbol{e}$ positions contain charged amino acids at the following frequency: arginine $(\mathrm{R}), 14 \%$; lysine $(\mathrm{K}), 18 \%$; aspartic acid $(\mathrm{D}), 1 \%$; and glutamic acid (E), 34\%. There are approximately equal numbers of basic and acidic amino acids in these two positions. It is notable that aspartic acid is not observed often in the $\boldsymbol{g}$ or $\boldsymbol{e}$ position of the coiled-coil structure, and model building suggests that the aspartic acid side chain is too short to form an optimal interhelical salt bridge.

The dimerization sequences presented contain about eight $\alpha$-helical turns or four $i+5$ interhelical electro- static interactions. We refer to these four potential $i+5$ interactions as the first, second, third, and fourth $i+5$ boxes, reading from left to right (the first salt bridge is closest to the DNA-binding region). If both the $\boldsymbol{g}$ and the following $\boldsymbol{e}$ amino acid are charged, the $\mathrm{i}+5$ boxes are color coded (36 of 80, Fig. 2); if only the $\boldsymbol{g}$ or the $\boldsymbol{e}$ position is charged, the $i+5$ boxes are not colored and are referred to as incomplete boxes ( 36 of 80 ), which will be discussed later. We have not color coded the incomplete boxes, which are observed as frequently as complete boxes for the simplicity of presentation, but we argue that they are important for determining heterodimer specificity. Only eight boxes do not contain a charged amino acid on either the $\boldsymbol{g}$ or $\boldsymbol{e}$ position. The color-coded $i+5$ boxes are classified into four groups according to the electrostatic charges in the $\boldsymbol{g}$ and $\boldsymbol{e}$ positions. A yellow box indicates that the $g$ position is acidic and the following $\boldsymbol{e}$ position is basic (acidic-xxxx-basic: yellow). The four $x$ s represent the four amino acids between the $g$ and $\boldsymbol{e}$ positions. When two helices with yellow boxes dimerize, the acidic and basic residues on opposite helices interact $\left(\boldsymbol{g}\right.$ interacts with $\boldsymbol{e}^{\prime}$ and $\boldsymbol{e}$ interacts with $\boldsymbol{g}^{\prime}$; see Fig. 1) to create two salt bridges. The two helices can be either identical or not, resulting in homodimer or heterodimer formation, respectively. Therefore, the interac-
LEUCINE ZIPPER REGION

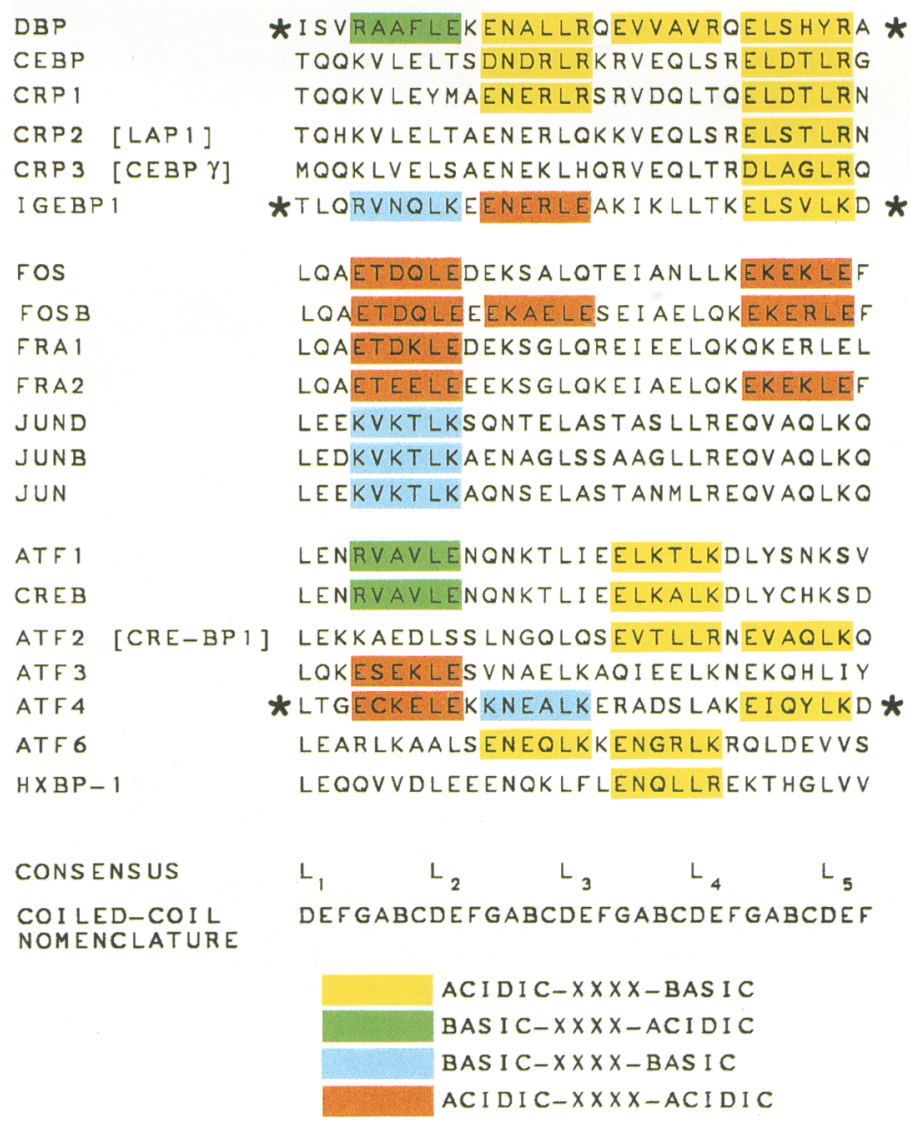

Figure 2. Amino acid sequences of the dimerization region of 20 mammalian bZIP proteins. Proteins are identified in the left-hand column. Polypeptide sequences designated by the single-letter code are aligned with respect to an invariant asparagine and arginine in the basic region just amino-terminal of the first $\boldsymbol{d}$ position (typically leucine) in the dimerization region (Vinson et al. 1989). This $\boldsymbol{d}$ position is the first amino acid in the sequences presented. The sequences have been grouped into three categories on the basis of their DNA-binding properties. Presumed interhelical salt bridge interactions are color coded. If the amino acid at the $\boldsymbol{g}$ position and the amino acid at the following $e$ position $(i+5)$ are both charged amino acids, the sequence from $g$ to $e$ is highlighted in a ATF (CREB) colored rectangle. As described in the text, FAMI LY within the same column, yellow can interact with yellow, green can interact with green, and blue can interact with red. The coiled-coil nomenclature is shown at the bottom. Amino acid sequences were obtained with the following sources: DBP (Wuarin and Schibler 1990); C/EBP, JunB, Fos, and CREB (Vinson et al. 1989); CRP1, CRP2 (LAP1 or C/EBP $\beta$ ), CRP3 (C/EBP $\gamma$ ) (Williams et al. 1991); IGEBPI C (Roman et al. 1990); FosB, Fral, Fra2, JunD, JunB, HXBP-1 (Kerppola and Curran 1991); ATF1, ATF2, ATF3, ATF4, ATF6 (Hai et al. 1989). 
tion between two yellow boxes would not selectively favor either homodimer or heterodimer formation. A green box indicates that the $g$ position is basic and the following $e$ position is acidic (basic-xxxx-acidic: green). Analogous to the yellow boxes, when two helices with green boxes dimerize, two salt bridges are created. We refer to these yellow-yellow and green-green interactions as self-complementary. We have no explanation for the observed preference of acidic-xxxx-basic pairs (yellow box: 16 pairs) over basic-xxxx-acid pairs (green box: 3 pairs). Because of the electrostatic repulsion between similar charges, yellow-green pairs and green-yellow pairs would disfavor dimerization.

The blue and red boxes indicate that the $\mathbf{g}$ and the following $e$ positions have a similar charge: They are either both basic (basic-xxxx-basic: blue) or both acidic (acidic-xxxx-acidic: red). When two helices with blue boxes interact, the basic $\boldsymbol{g}$ residue from one helix interacts with the basic $\boldsymbol{e}^{\prime}$ residue from the opposite helix, creating an electrostatic repulsion that discourages dimerization. The same holds true for two helices containing red boxes. However, if a helix containing a blue box and a helix containing a red box interact, then the basic charges in the blue box interact with the acidic charges in the red box to form two salt bridges. The bluered and red-blue interactions, which favor the formation of heterodimers, are referred to as mutually complementary. This combination is abundant in the Fos/Jun family, as other investigators have noted (Nicklin and Casari 1991; Schuermann et al. 1991; O'Shea et al. 1992).

This view of dimer specificity explains the limited bZIP dimerization data. All of the C/EBP proteins can heterodimerize (Roman et al. 1990; Cao et al. 1991; Williams et al. 1991), and all have the same type of selfcomplementary salt bridge, a yellow $i+5$ box in the fourth position. All of the Fos proteins have acidic amino acids (a red box) in the first $i+5$ position, whereas their natural partners, the Jun proteins, have basic amino acids (a blue box) in this position. These molecules heterodimerize (for review, see Abel and Maniatis 1989; Kouzarides and Ziff 1989b; Busch and Sassone-Corsi 1990; Kerppola and Curran 1991), presumably through these mutually complementary interactions.

\section{Prediction of novel bZIP partners}

To test the $i+5$ salt bridge rule in controlling dimerization specificity, we tried to predict novel dimer partners among the molecules listed in Figure 2. We predicted that IGEBP1 and ATF4 would heterodimerize preferentially. Both molecules have three colored i+5 boxes (first, second, and fourth) and are known individually to homodimerize (Hai et al. 1989; Roman et al. 1990). According to our color-coding system, ATF4 is red/blue/ uncolored/yellow and IGEBP1 is blue/red/uncolored/ yellow. Because blue and red are mutually complementary and yellow is self-complementary, the ATF4/ IGEBP1 heterodimer could form a total of three pairs of salt bridges and would contain fewer repulsive electrostatic interactions. Dimer specificity would not be de- termined by the salt bridges between the fourth $i+5$ boxes (yellow/yellow) as they do not favor either homodimer or heterodimer formation. The salt bridges between the first and the second $i+5$ boxes, however, do favor the ATF4/IGEBP1 heterodimer over either homodimer. A quantification of the presumed electrostatic interactions between these two proteins is indicated in Table 1. To test this prediction, we used the gel mobility-shift assay (Fried and Crothers 1981) to monitor heterodimer formation (Hope and Struhl 1987) between ATF4 and IGEBP1. This assay involves mixing proteins of different molecular weights and assaying for DNA binding. Because the proteins have different molecular weights, they form DNA-protein complexes of different mobilities. If a heterodimer forms between these two

Table 1. Interhelical salt bridges

\begin{tabular}{|c|c|c|c|c|c|c|}
\hline & \multicolumn{2}{|c|}{ Homodimer } & \multicolumn{2}{|c|}{$\begin{array}{l}\text { Heterodimer } \\
\text { with ATF4 }\end{array}$} & \multicolumn{2}{|c|}{$\begin{array}{l}\text { Heterodimer } \\
\text { with IGEBP1 }\end{array}$} \\
\hline & $1+$ & -1 & $1+$ & -1 & $1+$ & -1 \\
\hline \multicolumn{7}{|c|}{ A. AT4 and C/EBP family } \\
\hline ATF4 & 2 & 4 & & & & \\
\hline IGEBP1 & 2 & 4 & 6 & 0 & & \\
\hline DBP & 8 & 0 & 4 & 2 & 4 & 2 \\
\hline CEBP & 4 & 0 & 4 & 1 & & \\
\hline CRP1 & 4 & 0 & 4 & 1 & & \\
\hline CRP2 & 4 & 0 & 4 & 1 & & \\
\hline
\end{tabular}

\section{B. C/EBP mutants and wild-type $C / E B P$}

\begin{tabular}{lccccccc}
\multicolumn{7}{c}{ Homodimer } & \multicolumn{2}{c}{ Heterodimer } \\
\multicolumn{7}{c}{ with CEBP } \\
& (+ & -1 & l+ & -1 & L/L & L/S & S/S \\
\hline CEBP & 4 & 0 & & & 22 & 59 & 19 \\
A & 2 & 4 & 3 & 1 & 03 & 78 & 20 \\
B & 2 & 2 & 5 & 0 & 06 & 94 & 00 \\
E & 4 & 4 & 6 & 0 & 01 & 98 & 01 \\
F & 2 & 6 & 5 & 1 & 00 & 99 & 01 \\
H & 6 & 0 & 5 & 0 & 19 & 56 & 24 \\
I & 6 & 0 & 5 & 0 & 18 & 41 & 41 \\
J & 8 & 0 & 6 & 0 & 18 & 51 & 31 \\
K & 6 & 0 & 5 & 1 & 46 & 21 & 33 \\
L & 8 & 0 & 5 & 1 & 48 & 22 & 30 \\
\hline
\end{tabular}

(A) Number of presumed $\mathrm{i}+5$ interhelical interactions between ATF4 and members of the C/EBP family. The attractive and repulsive electrostatic interactions are designated + and - , respectively. The first two columns examine the homodimerization of each protein. The electrostatic interactions between C/EBP family members and ATF4 are in the third and fourth columns. The interaction between DBP and IGEBP1 are in the remaining two columns. $(B)$ Number of presumed $i+5$ interhelical electrostatic interactions between the C/EBP mutant homodimers and heterodimers with wild-type CEBP. Densitometric scans of the gel shifts from Fig. 7, a and b, were quantified and the total amount of DNA shifted was defined as $100 \%$. The relative amount of each complex is indicated on the right, the percentages for C/EBP are an average from Fig. 7 , a and $\mathrm{b}$. (L/L) Large homodimer; (L/S) heterodimer; (S/S) small homodimer. 
proteins, a DNA-protein complex of an intermediate mobility appears.

One difficulty in assaying the preference of heterodimer formation between IGEBP1 and ATF4 is that each of the homodimers binds a different DNA sequence. IGEBP1 binds C/EBP-type sites (ATTGC GCAAT), whereas ATF4 binds ATF/CREB sites (TGAC GTCA). To assay for the binding of heterodimers, we created a chimeric DNA-binding site containing a half dyad of C/EBP and a half dyad of ATF (TGAC GCAAT). We purified both IGEBP1 and ATF4 proteins (Fig. 3) and then examined the binding of equal moles of each protein alone and an equimolar mixture of both proteins to three different DNA sites: C/EBP, ATF, and the chimeric site. Figure $3 \mathrm{~b}$ shows that equal moles of either IGEBP1 or ATF4 homodimers bound to their own DNA sites with similar DNA-binding constants. However, ATF4 did not bind to the C/EBP site and IGEBP1 did not bind to the ATF site. When equal moles of the two protein molecules were mixed, the ATF4/IGEBP1 heterodimer formed and bound to all three DNA sequences. On the C/EBP and the chimeric site, the heterodimer was the only DNA-binding dimer (panels A and C). That heterodimers were preferred when equal moles of ATF4 and

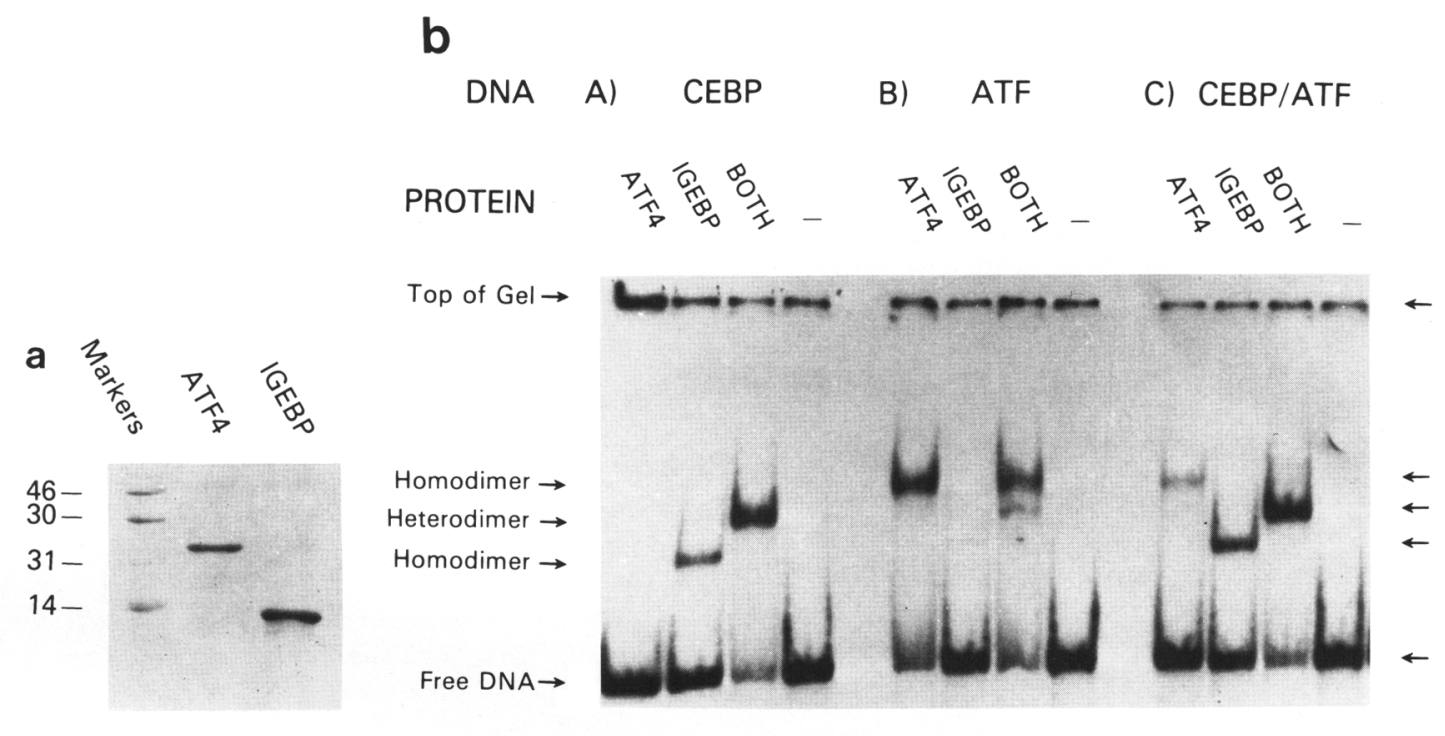

C

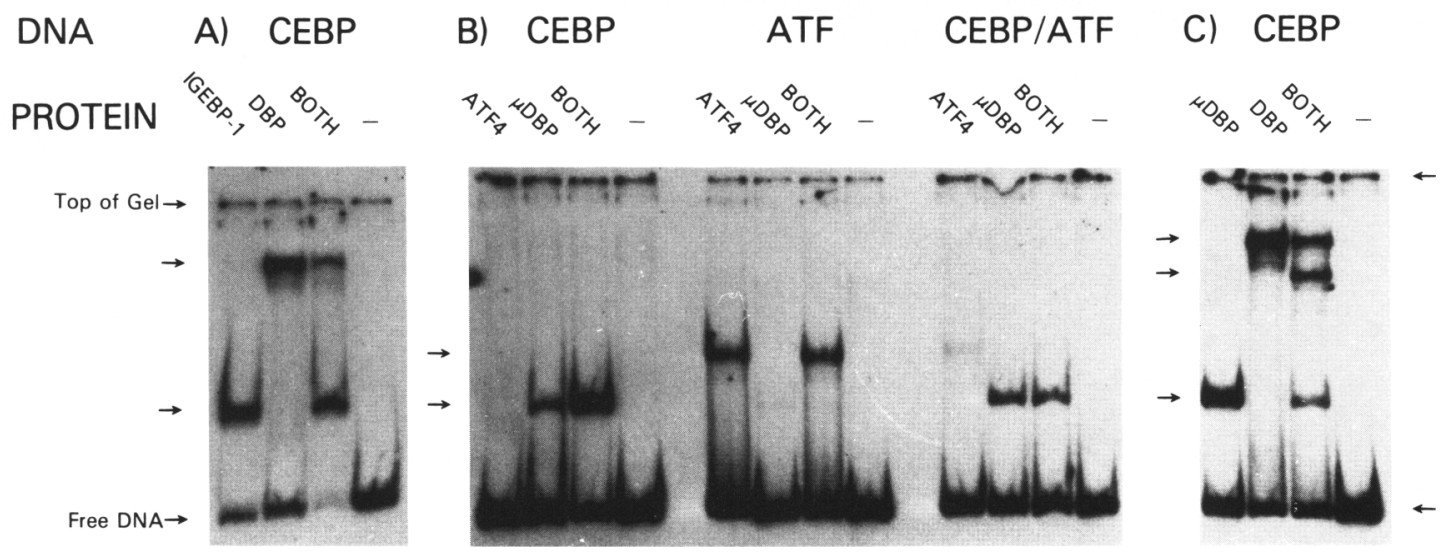

Figure 3. (a) Purified ATF4 and IGEBP1. We expressed and purified the bZIP regions of ATF4 and IGEBP1 from Escherichia coli to $95 \%$ homogeneity. Proteins were fractionated on a $20 \%$ SDS-polyacrylamide gel (Laemmli 1970) and stained with Coomassie blue. Protein concentrations were determined by the bicinchoninic acid method (Smith 1985) and used to determine molar concentration. (Lane 1) Size markers; (lane 2) ATF4; (lane 3) IGEBP1. (b) Dimerization of ATF4 with IGEBP1. Equal moles of ATF4 and IGEBP1, either alone or together, were bound to three different DNA sequences: (A) the C/EBP consensus, ATTGC GCAAT; (B) the ATF consensus, CTGAC GTCAG; and (C) the chimeric site containing a C/EBP and an ATF half site, CTGAC GCAAT. The space in the sequence denotes the center of the abutted dyad symmetric site. The lane marked both has twice as many molecules as the previous two lanes. The homodimers and heterodimer are indicated. (c) Dimerization of ATF4 or IGEBP1 with DBP (Iyer et al. 1991). (A) IGEBP1 and DBP, either alone or together, were incubated with the consensus C/EBP site. (B) ATF4 and DBP, either alone or together, were incubated with three different DNA sequences: C/EBP, ATF, and the chimeric site. (C) Two DBP molecules of different molecular weights, either alone or together, were incubated with the C/EBP site. The homodimer and heterodimer are indicated. 
IGEBP1 were mixed even on the C/EBP DNA-binding site indicates that heterodimer formation was not driven by the different DNA-binding properties of the heterodimer but by the greater stability of the heterodimer in the absence of DNA. This result demonstrates that the simple $i+5$ salt bridge rule is of value in predicting dimerization specificity.

We then attempted to predict a bZIP protein that would not form heterodimers with either ATF4 or IGEBP1. Examination of Figure 2 indicates that only DBP, a protein that binds the C/EBP site, has all of the possible $\mathrm{i}+5$ salt bridges occupied by self-complementary boxes (green and yellow). Therefore, we expected that DBP would preferentially homodimerize and would not form heterodimers with either ATF4 or IGEBP1. The results of mixing DBP with either IGEBP1 or ATF4 are shown in Figure 3c. As expected, DBP did not form heterodimers with either IGEBP1 or ATF4. As a control to prove that DBP could form heterodimers, two DBP derivatives with the same dimerization regions but different molecular weights were mixed and shown to heterodimerize. Again, the $i+5$ salt bridge rule predicted dimerization specificity.

Calame and colleagues (Roman et al. 1990) have shown that IGEBP1 can form heterodimers with other members of the C/EBP family. Knowing that ATF4 prefers to form heterodimers with IGEBP1, we examined whether ATF4 could also form heterodimers with other members of the C/EBP family. The numbers of presumed interhelical electrostatic interactions for each C/EBP homodimer and the respective heterodimer with ATF4 are similar and are shown in Table 1. As shown in
Figure 4, ATF4 forms heterodimers with the three C/EBP family members tested.

\section{Design of novel bZIP partners}

A problem with our predictions of novel dimerization partners is that those molecules have different DNAbinding regions that may affect our experimental results. To map definitively the dimerization specificity to the leucine zipper region we decided to create novel bZIP molecules with selected dimerization properties by altering the $i+5$ boxes. These created molecules would have identical basic regions but different leucine zippers. We started with the bZIP protein C/EBP and designed two classes of derivatives: (1) molecules that prefer to homodimerize and, therefore, do not heterodimerize with C/EBP, and (2) molecules that prefer to heterodimerize with C/EBP and, therefore, prevent C/EBP from homodimerizing. The latter class of molecules could be used as sophisticated dominant-negative molecules to disrupt the normal action of C/EBP in vivo.

We chose C/EBP as our starting molecule because it has two incomplete $i+5$ boxes: the first and third /see Fig. 2). By creating boxes in these positions that are either self-complementary or mutually complementary, we should be able to design proteins with desired dimerization specificities. For example, if we change the threonine in the $\boldsymbol{e}$ position of the first box to glutamic acid, we will convert this incomplete box to a self-complementary green box. This designed molecule would preferentially homodimerize rather than form a heterodimer with $\mathrm{C} / \mathrm{EBP}$ because the former event creates two salt

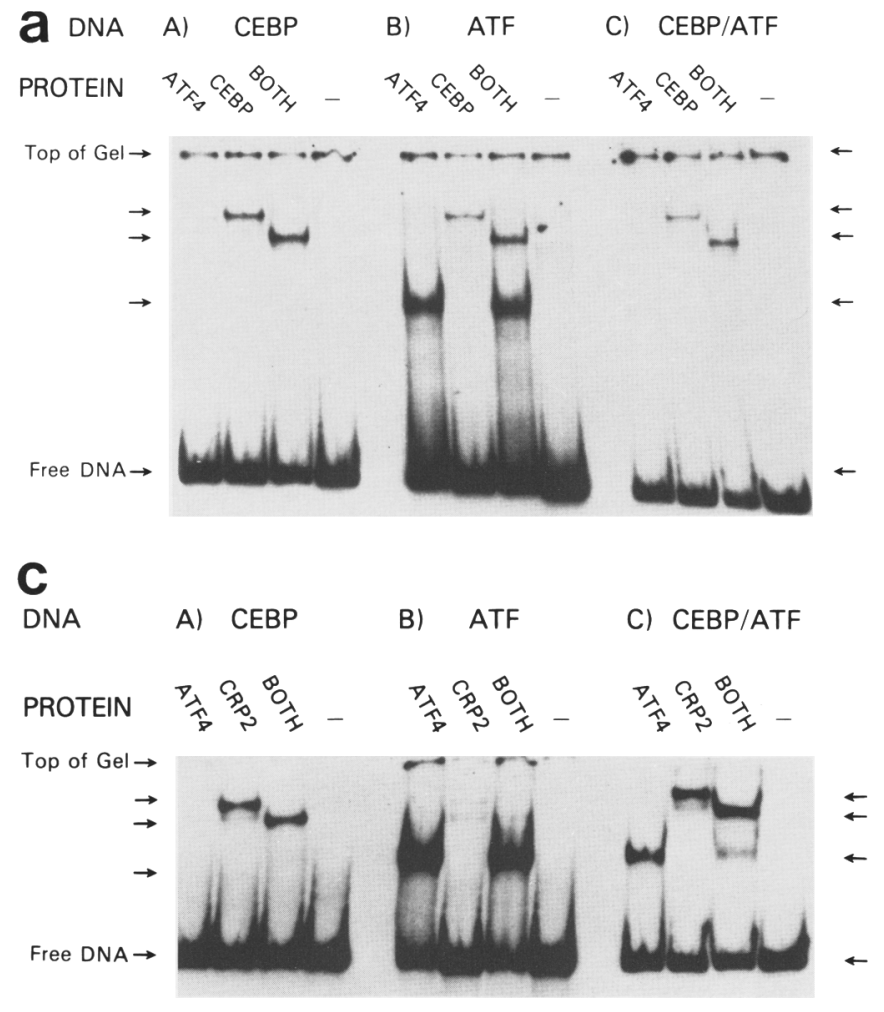

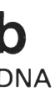

DNA

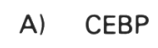

B) ATF

C) CEBP/ATF

PROTEIN

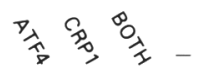

零象等

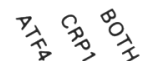

Top of $\mathrm{Gel} \rightarrow$

Free DNA $\rightarrow$
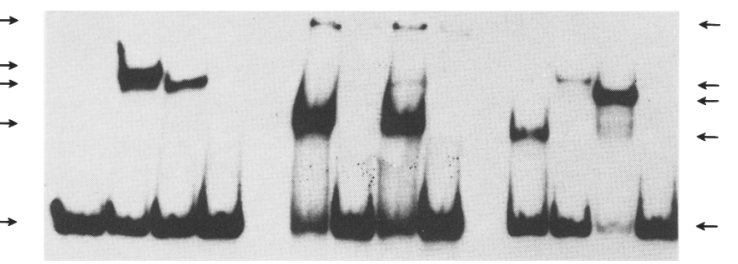

Figure 4. Dimerization of ATF4 with other members of the C/EBP family. (a) ATF4 and C/EBP either alone or together were bound to three different sequences detailed in the legend to Fig. 3. $(b)$ Same as $a$ except CRP1 was used instead of C/EBP. (c) Same as $a$ except CRP2 was used instead of C/EBP. 
bridges, whereas the latter event creates only one salt bridge as shown schematically in Figure 5.

Designed homodimers Figure 6A shows the five mutant protein sequences designed to homodimerize preferentially and, consequently, not heterodimerize with C/EBP. Both the first and third boxes of C/EBP contain a basic amino acid in the $\boldsymbol{g}$ position and an uncharged amino acid in the following $\boldsymbol{e}$ position. Mutant $\mathrm{H}$ optimizes the first salt bridge creating a lysine-glutamic acid salt bridge; mutant I optimizes the third salt bridge creating an arginine-glutamic acid salt bridge; and mutant $\mathrm{J}$ combines the mutations in $\mathrm{H}$ and $\mathrm{I}$. Because all three mutants, $\mathrm{H}, \mathrm{I}$, and $\mathrm{J}$, have incomplete $\mathrm{i}+5$ boxes converted into self-complementary boxes, as illustrated in Figure 5, they should prefer to homodimerize rather than heterodimerize with wild-type C/EBP. This prediction was tested by the gel-shift assay, and the results are shown in Figure 7A. The homodimeric and heterodimeric complexes were quantified by densitometric scans and are presented in Table 1. As predicted, mutants $\mathrm{H}, \mathrm{I}$, and $\mathrm{J}$ showed a modest and reproducible decrease in forming heterodimers with wild-type C/EBP. Combining mutants $\mathrm{H}$ and $\mathrm{I}$ to create mutant $\mathrm{J}$ did not create a better homodimerizing molecule.

To further test the $i+5$ salt bridge model, we inverted the amino acids in the third $i+5$ box by changing it from green to yellow in mutants $I$ and $J$ to create mutants $K$ and $L$, respectively. The net result of this conversion is

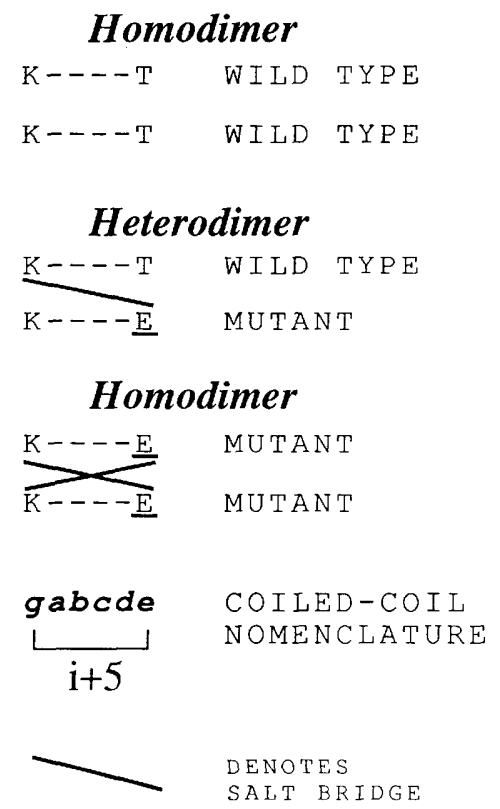

Figure 5. Consequence of changing 1 amino acid on potential salt bridge formation. The threonine $(T)$ in the $\boldsymbol{e}$ position has been changed to a glutamic acid (E) to produce an attractive $i+5$ interaction. The heterodimer between the wild-type and the designed protein has one additional salt bridge, whereas the homodimer between two designed proteins has two additional salt bridges. no change in the number or type of $i+5$ interactions (attractive vs. repulsive) when forming homodimers. However, when forming heterodimers with wild-type $\mathrm{C} / \mathrm{EBP}$, the $\mathrm{I} \rightarrow \mathrm{K}$ conversion results in one additional repulsive $i+5$ interaction; the $\mathrm{J} \rightarrow \mathrm{L}$ conversion results in not only one additional repulsive but also one fewer attractive $\mathrm{i}+5$ interaction (Table 1 ). As expected, $\mathrm{K}$ and $L$ heterodimerized less well with wild-type C/EBP than I and $\mathrm{J}$ (Fig. 7; Table 1).

Designed heterodimers The design of a protein that prefers to heterodimerize with wild-type C/EBP demands that heterodimerization is preferred over homodimerization of either protein. To drive heterodimer formation, we need to create additional salt bridges between the designed molecules and C/EBP. To discourage homodimer formation, we needed to ensure that repulsive interactions occur when the designed proteins form homodimers. Four C/EBP derivatives were created to fulfill these criteria (Fig. 6B) Mutant A creates an attractive heterodimer salt bridge in the first $\mathrm{i}+5$ box and repulsive electrostatic homodimer interactions in the first and second $i+5$ boxes. Mutant $B$ creates an attractive heterodimer salt bridge and a repulsive homodimer interaction in the third $\mathrm{i}+5$ box. Mutant $\mathrm{E}$ combines $\mathrm{A}$ and $\mathrm{B}$ while removing the repulsive second box homodimer interaction; mutant $\mathrm{F}$ is a combination of mutants $\mathrm{A}$ and B. A description of the $i+5$ electrostatic interactions between wild-type $\mathrm{C} / \mathrm{EBP}$ and mutant $\mathrm{F}$ is shown in Figure $6 \mathrm{C}$. Figure $7 \mathrm{~b}$ shows the results of gel-shift experiments. Both mutants A and B preferred to form heterodimers with C/EBP, although some homodimers remained. When these mutations were combined (mutant F), very little homodimer was formed, indicating that the mutations were additive. The number of potential attractive and repulsive $i+5$ interactions, along with a quantification of the gel shifts, is presented in Table 1.

Figure $7 \mathrm{c}$ shows the relative amounts of wild-type and mutant C/EBP protein used in Figure 7, a and b. Comparing the amount of protein used to the amount of DNA shifted /these quantities were determined by densitometric scans of both the protein gel and the gel-shift gel; data not shown), we did not observe a dramatic change in the ability of each of the nine designed proteins to bind DNA as homodimers. Thus, all of the changes that we have made in dimerization specificity have had no dramatic effect on DNA binding. The same result was obtained when the binding reactions were performed in the absence of dIdC (data not shown).

The small C/EBPs bind twice as well as the large C/EBPs (data not shown). This difference in DNA-binding constant demands that we add different moles of protein to get the same amount of DNA shifted. A concern in these experiments is that the apparent dimerization specificity may be the result of different molar quantities of small and large C/EBP in the same reaction. As a control, we performed experiments with large mutant proteins mixed with small wild-type C/EBP and vice versa. Figure $7 \mathrm{~d}$ shows the results of a gel-mobility assay in which wild-type C/EBP proteins and mutant $\mathrm{F}$ pro- 


\section{A}

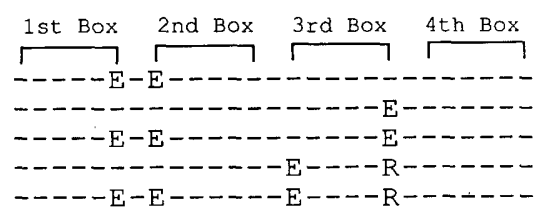

B

HETERODIMER

$\mathrm{E}----\mathrm{E}-\mathrm{R}---------------$

- - - - - - - - - - E-- - E-- - - -

E-D- $-\mathrm{E}--------\mathrm{E}----\mathrm{E}-------$

E--- E-R----- E--- $-\mathrm{E}-------$
A -1

B -3

E -6

$\mathrm{F}-4$
$\mathrm{C}$

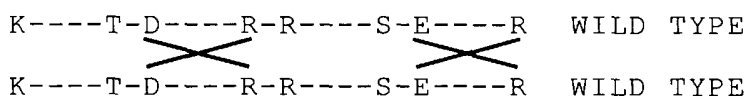

COLOR CODE

$\mathrm{G}-\mathrm{Y}-\mathrm{U}-\mathrm{Y}$

$U-Y-G-Y$

$G-Y-G-Y$

$U-Y-Y-Y$

$G-Y-Y-Y$

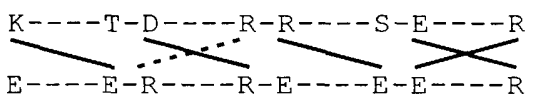

WILD TYPE

MUTANT (F)

E-- $-\mathrm{E}-\mathrm{R}---\mathrm{R}-\mathrm{E}---\mathrm{E}-\mathrm{E}---\mathrm{R} \quad \mathrm{MUTANT} \quad(\mathrm{F})$

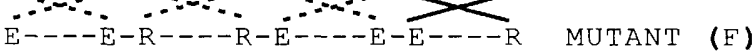

gabcdefgabcdef ${ }^{\star} g a b c d e f$ fgabcde ${ }^{*}$ COILED-COIL

NOMENCLATURE

$\mathrm{R}-\mathrm{B}-\mathrm{U}-\mathrm{Y}$

$\mathrm{U}-\mathrm{Y}-\mathrm{R}-\mathrm{Y}$

$R-Y-R-Y$

$\mathrm{R}-\mathrm{B}-\mathrm{R}-\mathrm{Y}$

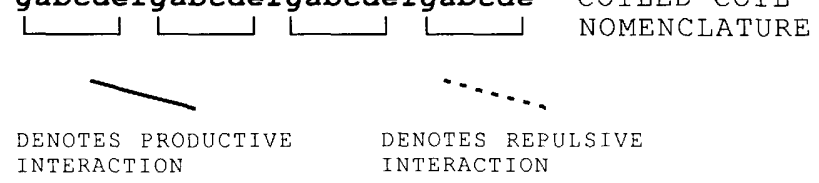

Figure 6. (A) Sequence of five C/EBP mutants designed to preferentially form homodimers with wild-type C/EBP. Only the mutated amino acids are shown. The letter designation of each designed protein is to the right of the sequence. The net change in charge between wild type and the designed protein is to the right of the design mutant sequence. The color codes for each $\mathrm{i}+5$ box are indicated to the far right $(\mathrm{G})$ green; $(\mathrm{Y})$ yellow; $(\mathrm{B})$ blue; $(\mathrm{R})$ red; $(\mathrm{U})$ uncolored. $(B)$ Sequence of four C/EBP mutants designed to preferentially form heterodimers with wild-type C/EBP. The net change in charge and the color codes are indicated to the right of the design mutant sequence. $(C)$ Design of a dominant-negative bZIP protein. The top pair of sequences represents the wild-type C/EBP homodimer, with only the $\boldsymbol{e}$ and $\boldsymbol{g}$ amino acids shown. The second pair of sequences shows wild-type C/EBP interacting with the mutant $\mathrm{F}$. The three amino acids that were mutated to discourage mutant homodimer formation are underlined. The two amino acids that were mutated to encourage heterodimer formation with wild-type C/EBP are double underlined. The bottom pair of sequences shows the homodimerization of mutant F. Presumed salt bridges are indicated with a solid line and repulsive interhelical electrostatic interactions are indicated by a broken line. The bottom line shows the coiled-coil nomenclature with the $\boldsymbol{g}$ and $\boldsymbol{e}$ positions highlighted by asterisks (*).

teins were mixed. The first two panels of Figure $7 \mathrm{~d}$ show the control experiments: Either wild-type C/EBP or mutant $\mathrm{F}$ was able to form heterodimers with both a long and short version of its own dimerization region. A $1: 2: 1$ ratio of large homodimers/heterodimers small homodimers was observed, as would be expected if the molecules had no preference for dimerization partner. The third and fourth panels show the result of mixing wild-type $\mathrm{C} / \mathrm{EBP}$ with designed mutant $\mathrm{F}$ proteins at the same concentrations used in the first and second panels. Heterodimers were clearly preferred regardless of whether the long molecule was wild type or designed protein. Because the assay worked in both cases, we feel confident that we are examining dimerization specificity and not a phenomenon resulting from mass action driving the reaction.

Taken together, the results obtained for the nine designed molecules demonstrate that we could control the dimerization specificity of C/EBP by modifying the interhelical i+5 electrostatic interactions.

\section{Discussion}

We have used the presumed interhelical electrostatic interactions between the two $\alpha$-helices in the dimeric leucine zipper structure to predict and design novel dimerization partners. Using the bZIP class of proteins, we predicted that ATF4 would form heterodimers with IGEBP1 and that neither of these proteins would form heterodimers with DBP. We used these same presumed interhelical electrostatic interactions to design C/EBP derivatives that would not form heterodimers with C/EBP or would preferentially form heterodimers with C/EBP.

A calculation of presumed interhelical salt bridges of the known mammalian bZIP proteins indicated that ATF4 would heterodimerize preferentially with IGEBP1. This same calculation of interhelical salt bridges predicted that neither ATF4 nor IGEBP1 would form heterodimers with DBP. These predictions were tested and verified using a gel-shift assay. Our calculations of interhelical electrostatic interactions focused on the first four heptads of the leucine zipper. Proceeding amino-terminally, the amino acid at the next $\boldsymbol{e}$ position is highly conserved, being either glutamic acid or glutamine. We think this conservation is important for the mechanistic details of DNA binding and is not used to regulate dimerization specificity.

The observed interaction between ATF4 and IGEBP1 is the first demonstration that proteins from the ATF or CREB family can form heterodimers with proteins from the C/EBP family. Significantly, the ATF4/IGEBP1 heterodimer binds to the chimeric DNA site, a "non-dyad symmetric site." This brings up the possibility that there may be many other bZIP heterodimers that can bind to nondyad symmetric sites. The physiological importance of the ATF/IGEBP1 heterodimer is not clear at this moment. A thorough survey of their cellular localization 


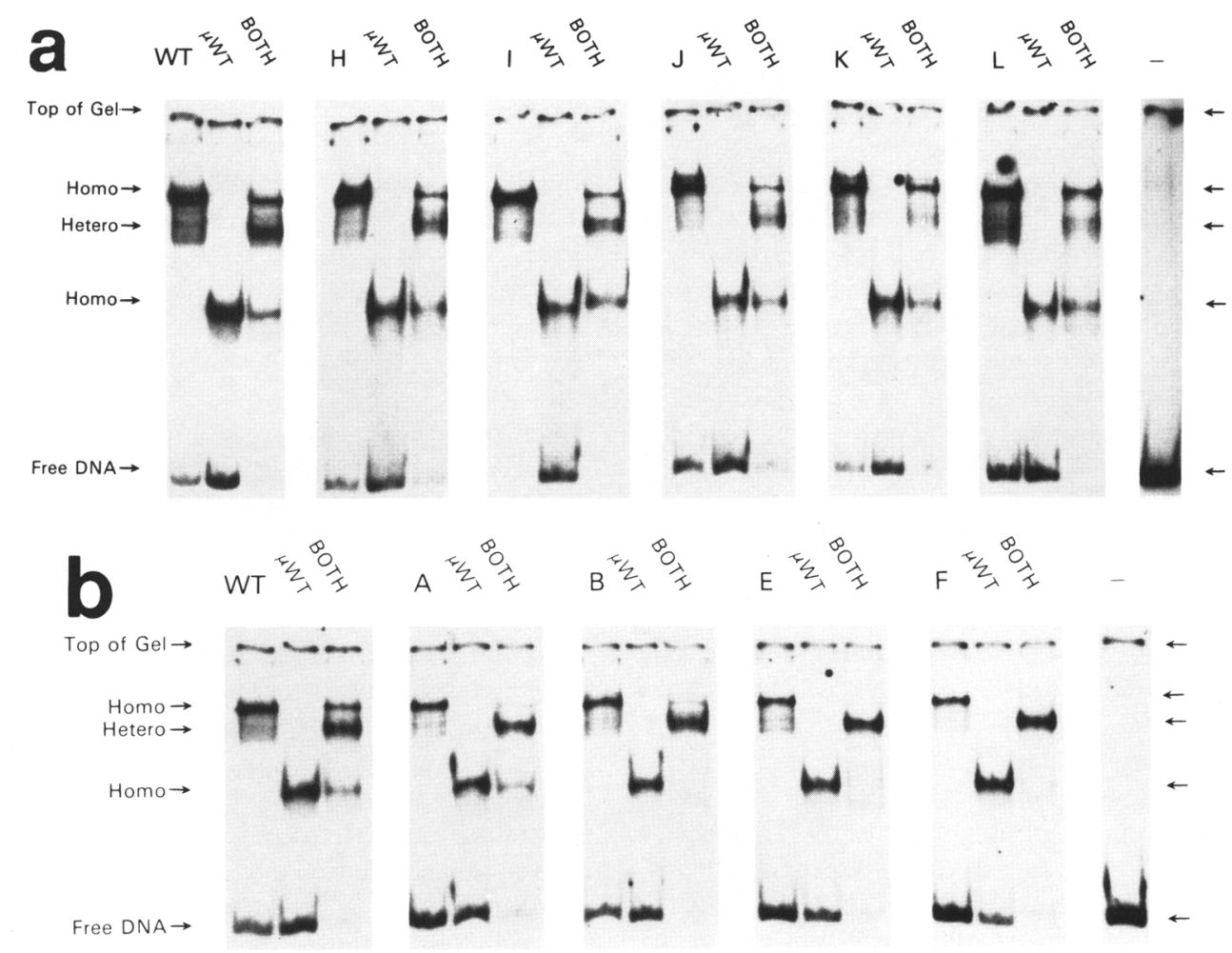

$C_{\text {A) }}$

B)

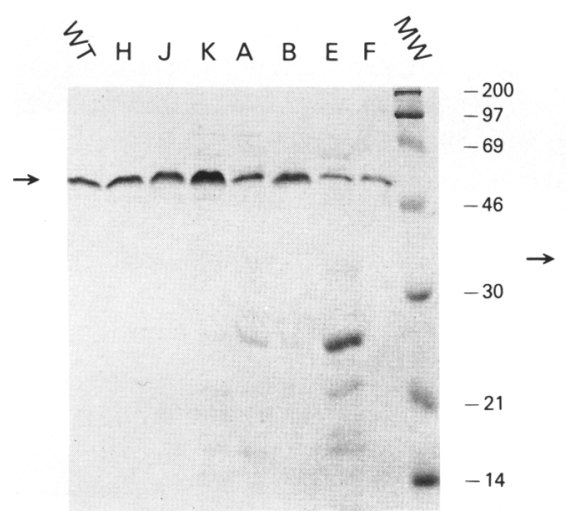

先弪

$-46$

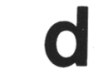

$\sum \xi \varepsilon_{\hat{\lambda}}^{\infty}$

$F \approx O_{i}^{\infty}$

$\sum_{\lambda} \varepsilon_{\lambda}^{\infty}$

$F \frac{\pi}{\lambda} \frac{\infty}{\frac{\lambda}{2}}$

Top of Gel $\rightarrow$
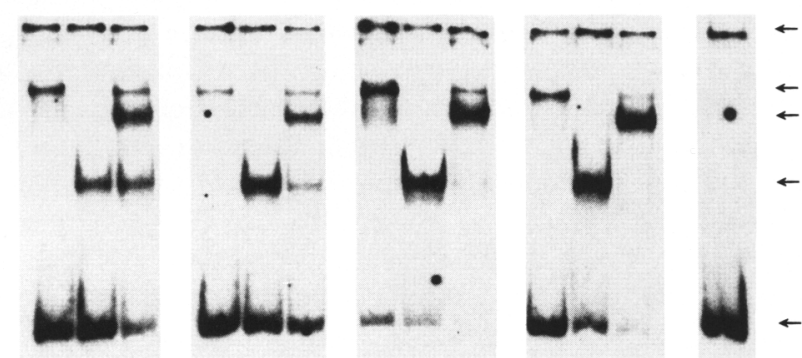

Figure 7. (b) Dimerization of wild-type C/EBP with mutants H, I, I, K, and L designed to homodimerize preferentially and not heterodimerize with wild-type C/EBP. A short wild-type C/EBP was mixed with the indicated mutant molecules before incubating with DNA containing the C/EBP site. In the six lanes labeled both, we tried to keep the amount of DNA shifted by each homodimer equivalent. This allows a visual examination of the abundance of the heterodimer. Note that the heterodimer is less abundant in all five mutants, particularly the last two, $\mathrm{K}$ and $\mathrm{L}$. $(b)$ Dimerization of wild-type C/EBP with mutants $\mathrm{A}, \mathrm{B}, \mathrm{E}$, and $\mathrm{F}$ designed to heterodimerize preferentially with wild-type C/EBP. A short wild-type C/EBP was mixed with the indicated mutant molecule before incubating with DNA containing the C/EBP site. The first panel shows long and short wild-type C/EBP, either alone or together. The lane labeled both shows the expected $1: 2: 1$ ratio of homodimers to herodimer if there was no preference for dimer partner. The next four panels show the A, B, E, and F mutants mixed with short C/EBP. Note that the heterodimer band becomes more prominent while the two homodimer bands disappear. (c) Protein concentration of C/EBP and the mutants. The same relative amount of each protein used in $a$ and $b$ was analyzed on a $10 \%$ SDS-polyacrylamide gel and stained with Coomassie blue. Note that the proteins, e.g., $\mathrm{E}$ and $\mathrm{F}$, were less abundant; and on the gel shifts shown in $b$, they also shifted less DNA. When the abundance of the proteins is compared with the amount of DNA shifted, there is less than a factor of 2 difference among all 10 molecules tested. (d) Dimerization of wild-type C/EBP with the designed mutant F. Long and short versions of both wild-type and mutant proteins were generated. (First panel, left). A long and short version of wild-type C/EBP, either alone or together, were mixed before incubating with DNA containing the C/EBP site. The homodimers and heterodimer are noted with arrows. The lane marked both shows an $\sim 1: 2: 1$ ratio of shifted bands, indicating that there is no preference for dimer formation. (Second panel) A long and a short version of designed mutant $F$, either alone or together, were mixed before incubating with DNA containing the C/EBP site. Again, note the $1: 2: 1$ ratio. (Third panel) A long version of wild-type C/EBP was mixed with a short version of designed mutant $F$. (Fourth panel) $A$ long designed mutant $F$ was mixed with a short version of wild-type C/EBP. Note that in the third and fourth panels, the heterodimer is the preferred band. 
will shed light on the possibility of forming this heterodimer in a biological system. The additional observation that ATF4 can form heterodimers, albeit with less specificity, with other members of the C/EBP family suggests that the biological possibilities of heterodimer formation on nondyad symmetric DNA sites will be numerous. We have performed additional experiments (data not shown) demonstrating that ATF3, a protein with less predicted heterodimer-forming potential with the C/EBP family, did form heterodimers with members of the C/EBP family, particularly on a chimeric site. As expected, the heterodimerization was less efficient than for ATF4.

Wild-type C/EBP and the nine mutant proteins all bound to DNA with similar affinities. This result was expected for those molecules designed to homodimerize preferentially and thought to be more stable dimers (mutants H, I, J, K, and L). Previously, we observed that the oxidation of a carboxy-terminal cysteine in wild-type $\mathrm{C} / \mathrm{EBP}$, which produces covalently bound dimers and are thus more stable, does not increase DNA binding (C.R. Vinson and S. McKnight, unpubl.). We know that the formation of more unstable C/EBP dimers produced by mutating the conserved leucines does disrupt DNA binding (Landschultz et al. 1989). The molecules designed to heterodimerize preferentially with C/EBP (mutants A, B, $\mathrm{E}$, and $\mathrm{F} /$ are imagined to produce less stable homodimers because of electrostatic repulsion. These proteins have amino acids in the $\boldsymbol{e}$ and $\boldsymbol{g}$ positions that are reminiscent of Fos proteins, which are unable to homodimerize. Figure 2 shows that of known mammalian bZIP proteins, only Fos proteins have three consecutive glutamic acids in the $\boldsymbol{e}$ and $\mathbf{g}$ positions. Investigators have shown that this arrangement is responsible for the inability of Fos to homodimerize (Nicklin and Casari 1991; Schuermann et al. 1991). One might have expected mutants B, E, and F proteins, which contain glutamic acids in three consecutive $\boldsymbol{e}$ and $\boldsymbol{g}$ positions, to bind to DNA less well than wild type. The fact that these mutants bind DNA similarly to wild-type C/EBP suggests that they are not so unstable as to affect DNA binding unlike the mutations to the conserved leucines. The reason why these molecules are still able to bind DNA, unlike Fos, needs additional study.

When comparing the nine C/EBP mutants, we do not observe a general correlation between electrostatic charge of a molecule and dimerization specificity. On the one hand, mutants $A$ and $B$ have a net charge change of -1 and -3 , respectively, and prefer to heterodimerize with wild-type C/EBP. On the other hand, mutants $\mathrm{K}$ and $L$ have a net charge change of -1 and -2 , respectively, and prefer to homodimerize rather than interact with wild-type C/EBP. Thus, overall electrostatic charge is not the rule that governs dimerization specificity; instead, it is the precise placement of the charged amino acids that is critical.

These data do not address whether attractive $i+5$ interactions, repulsive $i+5$ interactions, or both, are critical for determining dimerization specificity. Either interpretation fits our data well; to make heterodimerizing
C/EBP mutants, we have the homodimer with repulsive electrostatic interactions and the heterodimer with attractive electrostatic interactions. The homodimerizing C/EBP mutants are the only examples where the number of repulsive interactions are kept constant and the number of attractive interactions is varied. Increasing the number of attractive interactions (mutants $\mathrm{H}, \mathrm{I}$, and $\mathrm{J}$ ) has a modest effect, if any, on dimerization specificity, but the addition of one repulsive heterodimer interaction (mutants $\mathrm{K}$ and $\mathrm{L}$ ) drove the formation of homodimer. These results are consistent with the work of $\mathrm{O}^{\prime}$ Shea and colleagues (1992), who showed that Fos/Jun heterodimer formation is driven by the electrostatic repulsion of the Fos/Fos homodimer. The relevence of the presumed attractive $i+5$ interhelical electrostatic interactions is not demonstrated.

The results presented here show that the presumed i +5 electrostatic interactions can help in predicting dimer specificity as was observed in the predicted interaction between ATF4 and IGEBP1. This ability to predict dimerization partners will prove valuable as more bZIP proteins are identified in mammalian systems. It should be possible to predict dimer partners in genetic systems in which many bZIP proteins have been identified but little biochemical information is available. The ability to generate sophisticated dominant-negative molecules that specifically heterodimerize with native proteins, particularly if the DNA-binding specificity is changed, should be of interest in systems that do not have traditional genetics methods available, such as mammalian cell culture systems. These rules would be invaluable in the rational design of dominant-negative proteins and the subsequent inactivation of cellular gene products.

\section{Materials and methods}

\section{Proteins}

The ATF4 sample is a carboxy-terminal fragment of the native protein. The IGEBP1 sample includes only the bZIP motif. The C/EBP, CRP1, CRP2, DBP, and C/EBP mutant samples are chimeric proteins containing the amino terminus of GCN4 and the carboxyyl terminus of the C/EBPs, as described previously (Williams et al. 1991). The small versions of DBP and C/EBP contain less of the amino-terminal portion of the GCN4 protein.

\section{Protein expression and purification}

Proteins were synthesized in Escherichia coli using the phage T7 expression system (Studier and Moffatt 1986). Bacterial cultures $(400 \mathrm{ml})$ were induced with $1 \mathrm{mM}$ IPTG (isopropyl- $b$-Dthiogalactopyranoside) at an optical density of 0.6 at $600 \mathrm{~nm}$ for $2 \mathrm{hr}$. Cells were recovered by centrifugation, resuspended in 6 $\mathrm{ml}$ of lysis buffer [50 mM Tris- $\mathrm{HCl}(\mathrm{pH} \mathrm{8.0)}, 1 \mathrm{~mm}$ EDTA, $1 \mathrm{~mm}$ benzamidine, $1 \mathrm{~mm}$ dithiothreitol (DTT), and $0.2 \mathrm{~mm}$ phenylmethysulfonyl fluoride (PMSF)l, frozen, thawed, and gently brought to $1 \mathrm{M} \mathrm{KCl}$ by the addition of $2 \mathrm{ml}$ of $4 \mathrm{M} \mathrm{KCl}$. The sample was centrifuged at 25,000 rpm in a Beckman T42 rotor, and the supernatant was isolated. The isolated supernatant was then heated to $65^{\circ} \mathrm{C}$ for $10 \mathrm{~min}$ and centrifuged, and the supernatant was isolated. The ATF4 and IGEBPl proteins were diluted to $100 \mathrm{mM} \mathrm{KCl}$ and loaded onto a heparin-agarose col- 
umn. The column was washed with lysis buffer containing 100 $\mathrm{mM} \mathrm{KCl}$, followed by a $300 \mathrm{mM} \mathrm{KCl}$ wash, and eluted with buffer containing $1 \mathrm{M} \mathrm{KCl}$.

\section{Construction of mutant proteins}

Amino acid substitution mutants were introduced into C/EBP by the four-primer PCR mutagenesis method (Ho et al. 1989). DNA sequencing was performed on double-stranded templates using the Sanger dideoxynucleotide method (Sanger et al. 1977).

\section{DNA probe}

Oligonucleotides containing the three sequences presented in Figure $3 \mathrm{~b}$ were synthesized in the context of the sequence $5^{\prime}$ GACTGNNNNNNNNNNACTGA-3', annealed, and inserted into the SmaI site of pBluescript KS + plasmid polylinker. Each of the three probes was generated by digestion of the pBluescript plasmid with Asp718 (a neoschizomer of KpnI) and SacI. The digested DNA was treated with phosphatase to remove the $5^{\prime}$ phosphate, then phosphorylated with $\left[\gamma^{-32} \mathrm{P}\right] \mathrm{ATP}$ before the 120 -bp fragment was isolated.

\section{DNA-binding assay}

Proteins $(0.1-0.001 \mu \mathrm{l})$ were mixed in $20 \mu \mathrm{l}$ of the gel-shift reaction buffer [ $25 \mathrm{~mm}$ Tris (pH 8.0), $50 \mathrm{~mm} \mathrm{KCl}, 0.5 \mathrm{~mm}$ EDTA, $2.5 \mathrm{~mm} \mathrm{DTT}, 1 \mathrm{mg} / \mathrm{ml}$ of bovine serum albumin (BSA), $10 \%$ glycerol], heated to $65^{\circ} \mathrm{C}$ for $2 \mathrm{~min}$ to allow the molecules to mix, and cooled for $20 \mathrm{~min}$ to room temperature. Lanes labeled both contain the same amount of protein used in each of the single protein lanes explaining why more DNA is shifted. For the mutant C/EBP experiments, the amount of protein used in the reactions was adjusted to have similar amount of homodimer remaining in the both lane. Labeled probe $(0.6 \mathrm{ng})$ and dIdC $(0.5 \mu \mathrm{g})$ were added to the reaction. The samples were analyzed on a $5 \%$ polyacrylamide gel in $0.5 \times \mathrm{TBE}$ at room temperature.

\section{Acknowledgments}

We thank John Burch for initial conversations about DBP and interhelical salt bridges. We thank Kathryn Calame and Steve Artandi for the IGEBP1 construct and John Burch, Simon Williams, and Peter Johnson for the DBP, C/EBP, CRP1, and CRP2 constructs. We are grateful to Jon Shuman for his advice in purifying the different protein molecules and Mark Ryan and Bryant Lee for running the ATF4-IGEBP1 protein gel. We thank Valda Kilfoil, Alan Wolffe, and Andreas Baxevanis, and an anonymous reviewer for comments on the manuscript.

The publication costs of this article were defrayed in part by payment of page charges. This article must therefore be hereby marked "advertisement" in accordance with 18 USC section 1734 solely to indicate this fact.

\section{References}

Abel, T. and T. Maniatis. 1989. Action of leucine zippers. Nature 341: 24-25.

Agre, P., P. Johnson, and S. McKnight. 1989. Cognate DNA binding specificity retained after leucine zipper exchange between GCN4 and C/EBP. Science 246: 922-925.

Busch, S. and P. Sassone-Corsi. 1990. Dimers, leucine zippers and DNA-binding domain. Trends Genet. 6: 36-40.

Cao, Z., R. Umek, and S. McKnight. 1991. Regulated expression of three C/EBP isoforms during adiposide conversion of 3T3-
L1 cells. Genes \& Dev. 5: 1538-1552.

Cohen, C. and D. Parry. 1990. A-helical coiled coils and bundles: How to design an $\alpha$-helical protein. Protein 7: 1-14.

Ellenberger, T., C. Brandl, K. Struhl, and S. Harrison. 1992. The GCN4 basic region leucine zipper binds DNA as a dimer of uninterrupted $\alpha$ helices: Crystal structure of the proteinDNA complex. Cell 71: 1223-1237.

Fried, M. and D. Crothers. 1981. Equilibria and kinetics of lac repressor-operator interactions by polyacrylamide gel electrophoresis. Nucleic Acids Res. 9: 6505-6525.

Gentz, R., F.J. Rauscher III, C. Abate, and T. Curran. 1989. Parallel association of Fos and Jun leucine zippers juxtaposes DNA binding domains. Science 243: 1695-1699.

Hai, T. and T. Curran. 1991. Cross-family dimerization of transcription factors Fos/Jun and ATF/CREB alters DNA binding specificity. Proc. Natl. Acad. Sci. 88: 3720-3724.

Hai, T., F. Liu, W. Coukos, and M. Green. 1989. Transcription factor ATF cDNA clones: An extensive family of leucine zipper proteins able to selectively form DNA-binding heterodimers. Genes \& Dev. 3: 2083-2090.

Ho, S., H. Hunt, R. Horton, J. Pullen, and L. Pease. 1989. Sitedirected mutagenesis by overlap extention using the polymerase chain reaction. Gene 77: 51-59.

Hodges, R., J. Sodak, L. Smillie, and L. Jurasek. 1972. Tropomyosin: Amino acid sequence and coiled-coil structure. Cold Spring Harbor Symp. Quant. Biol. 37: 299-310.

Hope, I. and K. Struhl. 1987. GCN4, a eukaryotic transcriptional activator protein, binds as a dimer to target DNA. EMBO $J$. 6: 2781-2784.

Hu, J., E. O'Shea, P. Kim, and R. Sauer. 1990. Sequence requirements for coiled-coils: Analysis with $\lambda$ repressor-GCN4 leucine zipper fusions. Science 250: 1400-1403.

Ivashkiv, L.B., H.-C. Liou, C.J. Kara, W.W. Lamph, I.M. Verma, and L.H. Glimcher. 1990. mXBP/CRE-BP2 and c-Jun form a complex which binds to the cyclic AMP, but not to the 12O-tetradecanoylphorbol-13-acetate, response element. Mol. Cell. Biol. 10: 1609-1621.

Iyer, S., D. Davis, S. Seal, and J. Burch. 1991. Chicken vitellogenin gene-binding protein, a leucine zipper transcription factor that binds to an important control element in the chicken vitellogenin II promoter, is related to rat DBP. Mol. Cell. Biol. 11: 4863-4875.

Kerppola, T. and T. Curran. 1991. Transcription factor interactions: Basics on zippers. Curr. Opin. Struct. Biol. 1: 71-79.

Kouzarides, T. and E. Ziff. 1989a. Behind the Fos and Jun zipper. Cancer Cells 1: 71-76.

- 1989b. Leucine zippers of Fos, Jun and GCN4 dictate dimerization specificity and thereby control DNA binding. Nature 340: $568-571$.

Laemmli, U. 1970. Cleavage of structural protein during the assembly of the head of bacteriophage T4. Nature 277: 680685.

Landschultz, W., P. Johnson, and S. McKnight. 1988. The leucine zipper: A hypothetical structure common to a new class of DNA binding proteins. Science 240: 1759-1764.

- 1989. The DNA binding domain of the rat liver nuclear protein C/EBP is bipartite. Science 243: 1681-1688.

Marqusee, S. and R. Baldwin. 1987. Helix stabilization by Glu ... Lys salt bridges in short peptides of de novo design. Proc. Natl. Acad. Sci. 84: 8898-8902.

McLachlan, A. and M. Stewart. 1975. Tropomyosin coiled-coil interactions: Evidence for an unstaggered structure. J. Mol. Biol. 98: 293-304.

Nicklin, M. and G. Casari. 1991. A single site mutation in a truncated Fos protein allows it to interact with the TRE in vitro. Oncogene 6: 173-179. 
O'Neil, K., R. Hoess, and W. DeGrado. 1990. Design of DNA binding peptides based on the leucine zipper motif. Science 243: 774-778.

O'Shea, E., R. Rutkowski, and P. Kim. 1989a. Evidence that the leucine zipper is a coiled-coil. Science 243: 538-542.

O'Shea, E., R. Rutkowski, W.F. Stafford III, and P. Kim. 1989b. Preferential heterodimer formation by isolated leucine zippers from Fos and Jun. Science 245: 646-648.

O'Shea, E., J. Klemm, P. Kim, and T. Abler. 1991. X-ray structure of the GCN4 leucine zipper, a two-stranded, parallel, coiled-coil. Science 254: 539-544.

O'Shea, E., R. Rutkowski, and P. Kim. 1992. Mechanism of specificity in the fos-jun oncoprotein heterodimer. Cell 68: 699-708.

Roman, C., J. Platero, J. Shuman, and K. Calame. 1990. Ig/EBP1: A ubiquitously expressed immunoglobulin enhancer binding protein that is similar to C/EBP and heterodimerizes with C/EBP. Genes \& Dev. 4: 1404-1415.

Sanger, F., S. Nicklen, and A. Coulson. 1977. DNA sequencing with chain-terminating inhibitors. Proc. Natl. Acad. Sci. 74: 5463-5467.

Saudek, V., A. Pastore, M. Castiglione-Morelli, R. Frank, H. Gausepohl, and T. Gibson. 1991. The solution structure of a leucine-zipper motif peptide. Protein Engineering 4: 519529.

Schindler, U., A.E. Menkens, H. Beckman, J.R. Ecker, and A.R. Cashmore. 1992. Heterodimerization between the light-regulated ubiquitously expressed Arabidopsis GBF bZIP proteins. $E M B O$ /. 11: 1261-1273.

Schuermann, M., J. Hunter, G. Hennig, and R. Muller. 1991. Non-leucine residues in the leucine repeats of Fos and Jun contribute to the stability and determine the specificity of dimerization. Nucleic Acids Res. 19: 739-746.

Smith, P.K., R.I. Krohn, G.T. Hermanson, A.K. Mallia, F.H. Gartner, M.D. Provenszano, E.K. Fujimoto, N.M. Goeke, B.J. Olson, and D.C. Klenk. 1985. Measurement of protein using Bicinchoninic acid. Anal. Biochem. 150: 76-85.

Studier, F. and B. Moffatt. 1986. Use of bacteriophage T7 RNA polymerase to direct selective high-level expression of cloned genes. J. Mol. Biol. 189: 113-130.

Talanian, R., C. McKnight, and P. Kim. 1990. Sequence-specific DNA binding by a short peptide dimer. Science 249: 769771.

Turner, R. and R. Tjian. 1989. Leucine repeats and an adjacent DNA binding domain mediate the formation of functional cFos-cJun heterodimers. Science 243: 1689-1694.

Vinson, C., P. Sigler, and S. McKnight. 1989. A scissors-grip model for DNA recognition by a family of leucine zipper proteins. Science 246: 911-916.

Williams, S., C. Cantwell, and P. Johnson. 1991. Family of C/EBP-related proteins capable of forming covalently linked leucine zipper dimers in vitro. Genes \& Dev. 5: 1553-1567.

Wuarin, J. and U. Schibler. 1990. Expression of a liver-enriched transcriptional activator protein DBP follows a stringent circadian rhythm. Cell 63: 1257-1266. 


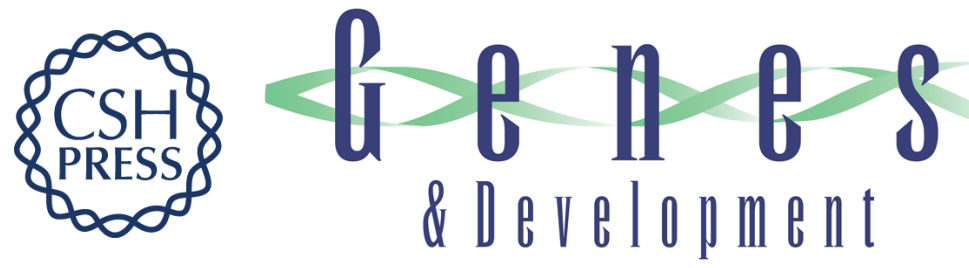

\section{Dimerization specificity of the leucine zipper-containing bZIP motif on DNA binding: prediction and rational design.}

C R Vinson, T Hai and S M Boyd

Genes Dev. 1993, 7:

Access the most recent version at doi:10.1101/gad.7.6.1047

References This article cites 42 articles, 20 of which can be accessed free at: http://genesdev.cshlp.org/content/7/6/1047.full.html\#ref-list-1

License

Email Alerting

Service

Receive free email alerts when new articles cite this article - sign up in the box at the top right corner of the article or click here.

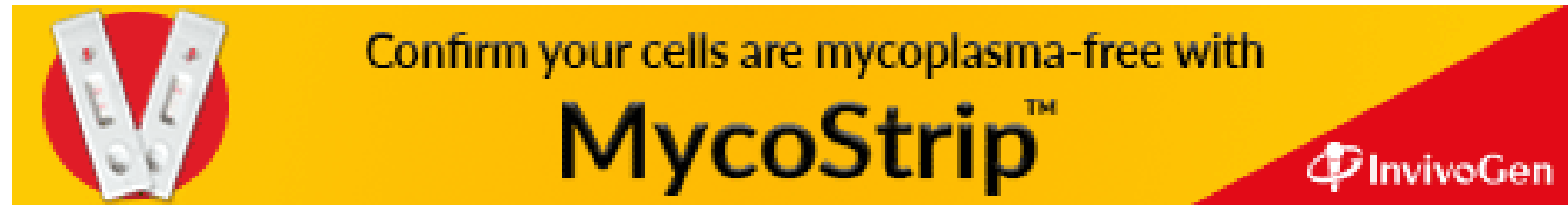

